\title{
Along-shore larval dispersal kernels in a numerical ocean model of the central Chilean coast
}

\author{
Christopher M. Aiken ${ }^{1,2}$, Sergio A. Navarrete ${ }^{1, *}$, Manuel I. Castillo1, \\ Juan Carlos Castilla ${ }^{1}$ \\ ${ }^{1}$ Estación Costera de Investigaciones Marinas, Las Cruces, and Center for Advanced Studies in Ecology and Biodiversity, \\ Pontificia Universidad Católica de Chile, Casilla 114-D, Santiago, Chile \\ ${ }^{2}$ Centro de Investigación en Ecosistemas de la Patagonia, Bilbao 449, Coyhaique, Chile
}

\begin{abstract}
Dispersal kernels provide a useful way to quantify the average spatial distribution of propagules originating from a given point in space. Consequently, dispersal kernels have been used in analytical and numerical studies of short- and long-distance dispersal of marine invertebrates and fish with pelagic larval stages. In most cases, the shape of dispersal kernels is pre-determined and parameterised with knowledge of larval duration or mean current velocities homogeneously across space. Here, the characteristics of planktonic larval dispersal for near-shore species in a realistic coastal ocean flow are investigated through the use of a numerical ocean model of a section of the central Chilean coast. The 3-dimensional primitive equation model was forced by 4 yr of observed winds from Las Cruces. Planktonic larval dispersal was simulated by advecting passive drifters using the evolving model velocity field. No a priori assumptions were made about diffusion-advection statistics. Drifters were released daily from regularly spaced locations along the coast and were considered to have settled if found within $1 \mathrm{~km}$ of the coast $30 \mathrm{~d}$ after release. Observed dispersal kernels were then calculated for each release location, and their variability in space and time was examined. This variability was found to be substantial over spatial scales less than a typical larval-advection scale, and, as a result, a spatially and temporally averaged dispersal kernel was inadequate as a global model of settlement. Large along-shore variation in the shape of dispersal kernels led to significant variation in the spatial pattern of connectivity among local sites, with some acting as net sources and some as net sinks within scales of 10s of kilometres. These results are linked to the alongshore and seasonal variability in ocean circulation, in particular close to shore. Both local and global dispersal kernels were found to be non-Gaussian, with their distribution related to that of the ocean velocity field. It is concluded that, in realistic flows with complicated coastal geometry, considerable departure from the expected Gaussian dispersal kernels based on homogeneous flow conditions can lead to complex spatial patterns of connectivity and successful settlement along a relatively simple but real coastline.
\end{abstract}

KEY WORDS: Larval settlement • Population connectivity • Recruitment • Marine reserves • Nearshore oceanography · Upwelling · Wind stress · Dispersal

\section{INTRODUCTION}

Many marine organisms possess a planktonic larval stage of development, during which their fate is dependent, among other factors, on the vagaries of ocean flow. Successful settlement requires that larvae reach a suitable habitat within a competency time window at the end of larval development; thus, it is strongly influenced by local oceanic advective and diffusive time scales. Since knowledge of the level of connectivity among sub-populations, as well as the specific locations of net sources and sinks of competent larvae, are vital in management and conservation of benthic species, estimation of oceanic larval dispersal is an important goal. Because of the small, usually microscopic size of most invertebrate larvae and the 
long time periods spent in the plankton, quantifying larval dispersal empirically is extremely difficult. While the use of highly variable genetic markers (Kinlan \& Gaines 2003) and elemental microchemistry or fingerprinting (Levin 2006) are invaluable tools to estimate dispersal distance and potential larval origin, characterising the fate of nearly microscopic larvae originating at a given site is virtually impossible. The study of larval dispersal and its consequences for the design and effectiveness of marine reserves has therefore largely relied on theoretical models. Most of these models assume some form of dispersal kernel (larval settlement probability distribution) of the targeted species as the starting point to examine the effects of advective and diffusive processes connecting local sub-populations (Botsford et al. 1994, Kaplan 2006). Here, we used a coupled physical-biological Lagrangian model to track the dispersal of larvae released near-shore and construct 'empirical' dispersal kernels after larval development. This approach allowed us to examine the magnitude of along-shore and temporal variations in dispersal kernels and the consequences of this variation for connectivity and spatial patterns of larval sources and sinks under a realistic circulation scenario. We kept the biological part of the model as simple as possible in an effort to examine the degree of spatio-temporal heterogeneity introduced by winddriven oceanic flows alone.

If variations in ocean currents are assumed to be well represented by a spatially homogenous stochastic noise process, then dispersal of passive tracers may be described by a Fokker-Planck equation:

$$
\frac{\partial P}{\partial t}=-U \frac{\partial P}{\partial x}+\frac{1}{2} \sigma^{2} \frac{\partial^{2} P}{\partial x^{2}}
$$

where $P(x)$ is the probability density at position $x, U$ is the mean ocean velocity, $\sigma^{2}$ is the variance of the stochastic noise process and $t$ is time. This equation has as its solution a Gaussian dispersal kernel, with the mean value given by $U t$ and the standard deviation given by $\sigma t^{1 / 2}$ (Gardener 1985). Such a distribution is an obvious null model of ocean dispersal under idealised homogenous conditions (e.g. Siegel et al. 2003). For the particular case of larval settlement on the coast, the dispersal kernel is simply the 1-dimensional Gaussian solution during the competency time period. Many studies of larval dispersal and of the design of marine reserve networks have this basic approximation at their core (Botsford et al. 1994, Gaines et al. 2003). The ability of a simple Gaussian dispersal kernel based on the long-term ocean velocity mean and variability to predict along-shore larval distributions is compromised, however, by the degree to which the ocean flow diverges from the assumptions made in Eq. (1), namely of spatial and temporal homogeneity and of the vari- ability being sufficiently uncorrelated over the larval dispersal time period. Empirical studies of real ocean flows have shown that the mean and variability can change greatly over larval advection length scales, both along- and cross-shore. Similarly, the presence of transient features such as headland eddies can provide retention mechanisms that are important for the final settlement distributions. In general, if the variability in the ocean circulation is correlated over time scales similar to those corresponding to larval advection, the white noise assumption in Eq. (1) will not be valid. It is then important to determine to what extent a dispersal kernel based on spatially averaged statistics over larval advection length scales can represent the essential aspects of larval dispersal and connectivity under typical conditions encountered in the coastal ocean.

Besides the far-reaching influence of dispersal on the biology, ecology and evolution of species (Kinlan \& Gaines 2003), characterising the shape of dispersal has deep practical implications for conservation biology. Indeed, changes to the assumed dispersal kernel and mean dispersal distance, as well as the relative influence of advective and diffusive processes, can drastically influence decisions on optimal spacing and size of protected areas because they will alter the degree of connectivity among local sub-populations and their capacity for self-replenishment (Gaines et al. 2003, Cowen et al. 2006, Kaplan 2006). Yet, studies of marine reserve design have often assumed spatially uniform dispersal that is either completely extensive or completely local, which is an unlikely scenario for most species (Gerber et al. 2003). Advection-diffusion models such as those presented by Roughgarden et al. (1988) provide a more realistic estimation of ocean dispersal, but often have been implemented with poorly chosen parameter values (Largier 2003). Correct estimation of oceanic advective and diffusive scales has been shown to be vital for determining the degree of connectivity between populations (Cowen et al. 2000, Largier 2003, Siegel et al. 2003). Appropriate means for estimating dispersal kernels from knowledge of ocean flow statistics have been investigated (e.g. Largier 2003, Siegel et al. 2003). While, as discussed above, simple spatially homogenous advection-diffusion models result in Gaussian kernels, in nature dispersal kernels are often found to be leptokurtic (e.g. Chesson \& Lee 2005). Consequently, a number of studies have investigated the effect of dispersal kernel shape upon marine reserve design under idealised conditions over constant or temporally variable advection (e.g. Lockwood et al. 2002, Kaplan 2006).

The continued improvement in coastal ocean modelling provides the possibility to simulate larval dispersal in 'realistic' flow fields, and thus presents a convenient means to try to reconcile ocean circulation and dispersal theory. Numerically generated flow fields have 
been used to examine larval distribution and settlement for several marine organisms (e.g. Roberts 1997, Cowen et al. 2000, 2006, Guizien et al. 2006). These models are usually finely tuned to species-specific larval behaviours and attempt to explain or predict their larval abundance or settlement patterns. Numerical particle-tracking techniques have also been used in a more general context by Siegel et al. (2003) for estimating dispersal kernels. Our strategy is similar to that of Siegel et al. (2003) in terms of using realistic velocity fields to estimate statistics of larval dispersal, but in this case realistic velocity fields from a high resolution numerical ocean model are used in place of idealised stochastic ocean velocity fields. Our goal is to quantify spatial and temporal variability in dispersal kernels and patterns of connectivity along a real eastern boundary coastline; therefore, we kept the biological part of the model as simple as possible in order to concentrate on the influence of ocean advection.

The numerical model simulates ocean circulation for a section of the central Chilean coast, centred on the Estación Costera de Investigaciones Marinas (ECIM) in Las Cruces (see LC in Fig. 1b), where regular surveys of invertebrate recruitment patterns were taken and oceanographic and meteorological data were collected. The central coast of Chile is one of the world's major upwelling ecosystems (Strub et al. 1998), and there is substantial meso-scale (10s of kilometres) variability in the intensity and general characteristics of coastal upwelling within the region (Wieters et al. 2003, Narváez et al. 2004). Our intent in the present study was 2-fold: (1) to investigate spatial and temporal variability in larval transport, settlement and realised dispersal kernels in this region; and (2) to examine the consequences of this variation on patterns of spatial connectivity and variation in larval sources and sinks. We focus on model species that release larvae near-shore, like many of the species exploited commercially by the intense Chilean artisanal fishery. We later discuss possible differences with species that release larvae further off-shore.

\section{MATERIALS AND METHODS}

Numerical circulation model. Time-varying velocity fields for the region of interest were simulated using a numerical coastal ocean model. The model used here was the Environmental Fluid Dynamics Code (EFDC; Hamrick 1992), which solves the 3-dimensional, hydrostatic, free-surface primitive equations on either curvilinear or orthogonal grids in the horizontal and sigma (or terrain following) levels in the vertical. Temperature and salinity evolve through coupled transport equations, and the system is closed with a standard modification of the Mellor-Yamada Level 2.5 turbulence scheme. Full details of the numeric calculations and model physics can be found in Hamrick (1992). The model is well tested and has been previously applied successfully in a wide range of studies.

The model domain (Fig. 1) covers the section of the Chilean central coast between ca. 32.5 and $34.5^{\circ} \mathrm{S}$, a
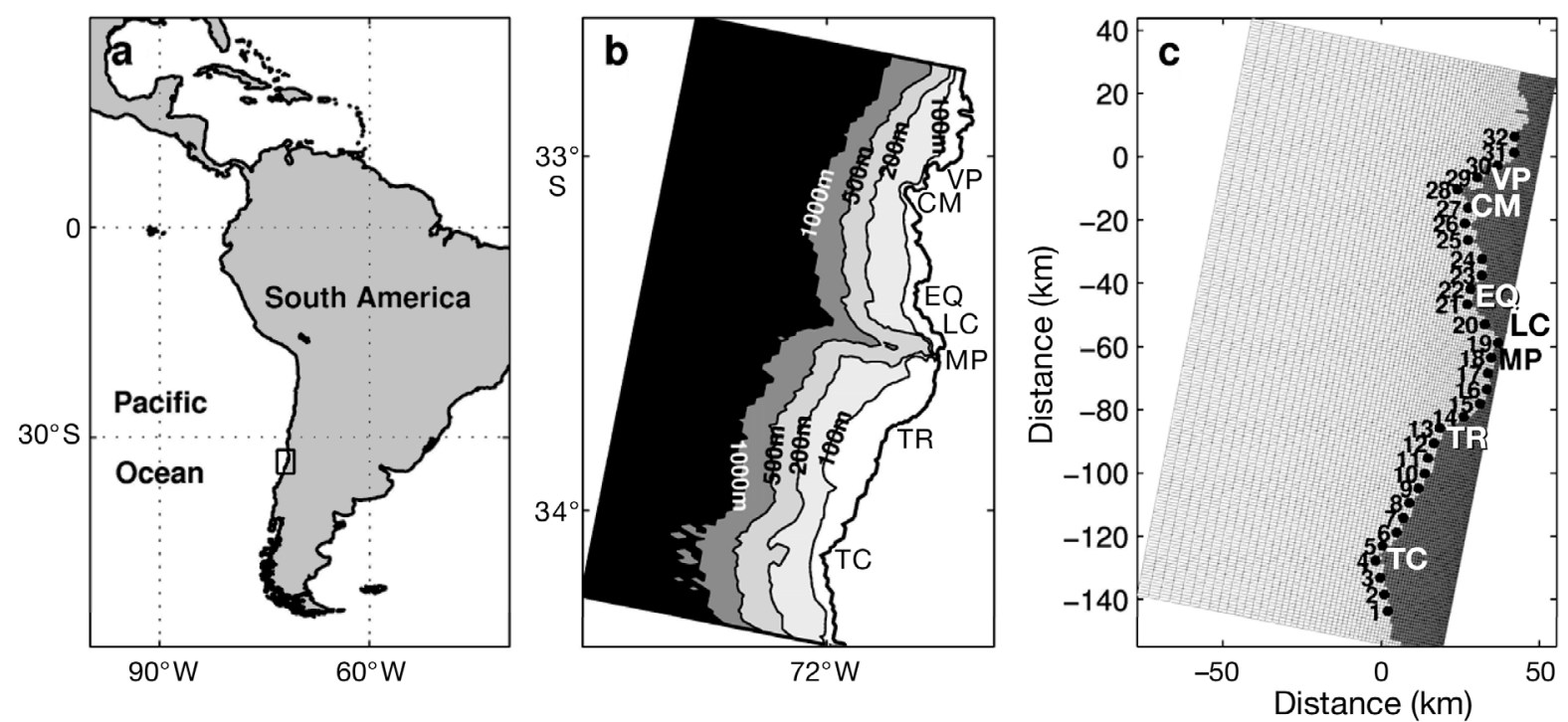

Fig. 1. (a) The South American west coast where the modelled region is indicated by the box. (b) Bathymetry of the modelled region. Depths are given in metres and the coastline marked as the thicker contour. Key locations are indicated: Valparaiso (VP), Curaumilla (CM), El Quisco (EQ), Las Cruces (LC), Maipo River mouth (MP), Punta Toro (TR) and Punta Topocalma (TC). (c) The model grid and release locations. The $x$ - and $y$-axes coordinates in Panel c are the distance in kilometres east and north, respectively, of $\left(33^{\circ} \mathrm{S}, 72^{\circ} \mathrm{W}\right)$. The average wind direction is from the south-west 
distance of approximately $200 \mathrm{~km}$, and extends $140 \mathrm{~km}$ off-shore. The modelled region is micro-tidal in dynamics, and currents are dominated by winds. An orthogonal grid was used (Fig. 1c), rotated clockwise by $11.25^{\circ}$ so as to follow the general orientation of the coast. This direction will be referred to hereafter as along-shore, and orthogonal to this, as cross-shore. Along-shore grid spacing was constant at $1 \mathrm{~km}$, while cross-shore grid spacing increased smoothly from $500 \mathrm{~m}$ next to the coast to $8 \mathrm{~km}$ at the off-shore edge of the domain. The high resolution of the model at the coast allowed good representation of the coastline and the bathymetry in shallow regions. An accurate representation of the bathymetry was found to be important for correctly reproducing certain features of the flow, such as the upwelling shadow typically observed at Las Cruces during the austral spring to summer months (Wieters et al. 2003, Narváez et al. 2004). The bathymetry of the region is characterised by its extremely narrow shelf and the presence of the Maipo submarine canyon at the mouth of the Maipo River (MP in Fig. 1b). Sigma-coordinate, density-stratified ocean models typically develop spurious currents over such steep bathymetry, a result of round-off error in the calculation of the pressure gradient on sigma surfaces. The problem was addressed in this study by smoothing the bathymetry and through the use of high horizontal resolution. As a result, under typical forcing, the magnitudes of erroneous currents were found to be negligible in all cases once the model was spun-up. The bathymetry was cropped at $1000 \mathrm{~m}$ depth, and 11 sigma levels were used, concentrated at the surface and bottom so as to maximise resolution of the respective boundary layers. The results that follow were found to be insensitive to a doubling of the number of vertical levels.

Boundary conditions at the alongshore ends of the numerical domain were periodic in all variables. A $15 \mathrm{~km}$ buffer region was added to each end of the model grid in which the bathymetry was linearly interpolated to provide a smooth transition across the periodic boundary. A periodic treatment of along-shore boundaries is a commonly used solution to the open boundary problem in the study of circulation driven by the local wind in regions with relatively straight coastlines. A condition of no normal flow was imposed at the off-shore boundary.

The model was forced predominantly by application of a time-dependent surface wind stress and to a lesser degree through relaxation to the climatological density profile, as discussed below. Daily wind stresses were derived from wind velocity recorded at ECIM in Las Cruces and then interpolated linearly to the model time-step. The wind observations are discussed in detail by Kaplan et al. (2003) and Narváez et al. (2004). The daily mean wind stress was estimated using winds from the period 13:00 to 16:00 h each day, the most reflective of the open ocean wind. The wind stress field was spatially homogenous, except inside Valparaiso Bay (VP in Fig. 1b), where, under southerly wind forcing, wind stress was reduced linearly to zero as a function of distance from the south-east corner of the bay. This is consistent with qualitative observations of Valparaiso Bay, which is well sheltered from the south, and was found to be important for correctly simulating the upwelling shadow in Valparaiso Bay (Lagos et al. 2005). More details of the importance of wind-sheltering for the circulation in Valparaiso Bay are given by C. Aiken et al. (unpubl. data). Winds in this region are from the southwest (upwelling favourable) for much of the year and intensify in austral spring and summer (Fig. 2). Downwelling-favourable winds occur briefly during austral winter, corresponding to the passage of atmospheric fronts and strong northerlies (Narváez et al. 2004).

The 3-dimensional temperature and salinity fields were initialised to climatological values from the NODC Levitus World Ocean Atlas 1998 (for 33 $\mathrm{S}$, $72^{\circ} \mathrm{W}$ ) at the initial month of simulation and were relaxed towards the appropriate climatology of the month via Newtonian damping with a relaxation time scale of $15 \mathrm{~d}$. This is a relatively weak relaxation that allows baroclinic structure to evolve freely. The Levitus data were provided by the NOAACIRES Climate Diagnostics Center, Boulder, Colora-

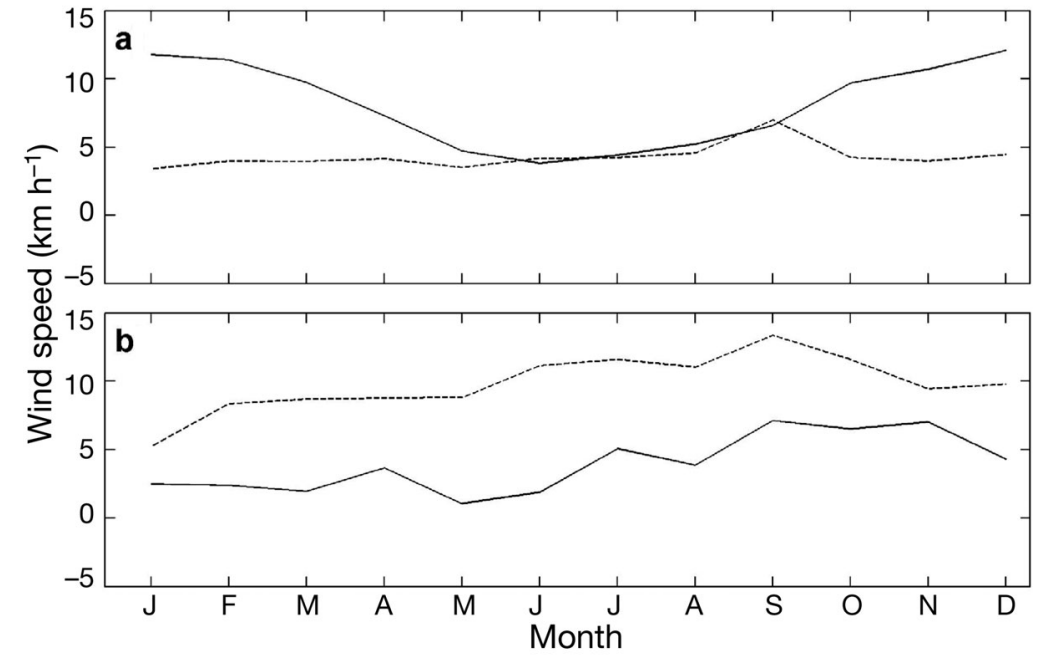

Fig. 2. (a) Monthly mean and (b) standard deviation in the eastwards (solid line) and northwards (dashed line) components of the $4 \mathrm{yr}$ wind time series from Las Cruces used to force the model 
do, USA, from their website at www.cdc.noaa.gov. The relaxation time scale was reduced linearly towards a value of $1 \mathrm{~d}$ over the width of the periodic buffer zones, in order to provide some damping of baroclinic structures re-entering the domain through the periodic boundary. Velocity and sea surface elevation were initialised to zero, and the model was spun-up for $30 \mathrm{~d}$ using the observed ECIM winds corresponding to November 1999. An intense upwelling event that occurred during this month is discussed by Poulin et al. (2002) and was well represented by the model. The model was then integrated forward for $1490 \mathrm{~d}$, corresponding to the period from 1 December 1999 to 1 January 2004.

The numerical model thus defined simulates the wind-driven velocity field for the region, but it must be noted that this is only one component of the velocity variability in the system. Contributions due to large-scale, along-shore pressure gradients, coastally trapped waves, the meso-scale eddy field, river plumes and tidal bores (e.g. Piñones et al. 2005, Narváez et al. 2006) may each be significant in this region at various times and are not included in the model. In addition, we did not parameterise velocity variability at scales finer than the model resolution, such as turbulence, which has been shown to have important consequences for dispersal in other areas (e.g. Guizien et al. 2006). Each of these processes would be expected to play a role in determining net larval dispersal; however, the importance of these is expected to be of secondary order, as the variability due to the local wind is known to be dominant in this region (Narváez et al. 2004, 2006). The overwhelming importance of wind forcing is confirmed in the model, which successfully simulates the major features observed in the region based on only the daily wind field (Aiken et al. unpubl. data). For instance, the few available direct ocean velocity observations in the modelled region (Narváez et al. 2004, Piñones et al. 2005) are generally consistent with the simulated velocity field. The phase and extent of upwelling events were successfully predicted, as determined from comparisons of modelled and satellite sea surface temperatures at Las Cruces and upwelling centres. Moreover, in the modelled flow an equatorward baroclinic jet is found above the shelf break for most of the year, intensifying during the upwelling season, weakening or occasionally reversing during autumn to winter, as is observed in both the California Current and Chile Coastal Current systems (Strub et al. 1998). Velocities within the jet reach up to $80 \mathrm{~cm} \mathrm{~s}^{-1}$ where the jet passes Curaumilla (CM in Fig. 1b), but otherwise are predominantly on the order of $10 \mathrm{~cm} \mathrm{~s}^{-1}$. In-shore of the jet, currents tend to be weak and variable. Major upwelling centres are located at Punta Topocalma (TC in Fig. 1b), El Quisco (EQ in Fig. 1b) and on the northern side of Curaumilla. Flow separation and eddy generation occurs sporadically north of Curaumilla and to a lesser extent north of Punta Toro (TR in Fig. 1b), usually upon relaxation after strong upwelling events (for more details see Aiken et al. unpubl. data).

Lagrangian larval dispersal model. To simulate the dispersal of the planktonic larvae of near-shore species, at the beginning of each simulated day a set of 100 Lagrangian drifters was released from each of 32 locations along the coast. The release locations, shown in Fig. 1c, were separated by $5 \mathrm{~km}$ along-shore. At each location the 100 drifters were initially spread evenly over a $1 \times 1 \mathrm{~km}$ area, centred $1 \mathrm{~km}$ off-shore and at a depth of approximately $5 \mathrm{~m}$. The 3-dimensional location of each drifter was updated at each time-step using a second-order accurate integration scheme with the same $180 \mathrm{~s}$ time-step as the circulation model. The particle-tracking algorithm prevented larvae from striking the coast by decreasing the timestep as necessary. Use of a higher order accurate scheme was found to provide little improvement in accuracy, but considerable increase in computational burden.

In order to isolate the influence of ocean currents alone, we modelled passive (non-swimming), neutrally buoyant larvae, which reach competency after $30 \mathrm{~d}$. Larval developments of 3 to $4 \mathrm{wk}$ are common to many benthic invertebrates (Kinlan \& Gaines 2003). Specific larval behaviours, ontogenetic changes in larval swimming velocities, ability to delay metamorphosis, and overall larval duration are known to alter the effects of oceanic flows on dispersal (e.g. Guizien et al. 2006). However, given that there is virtually no information on larval behaviour in this region, our approach here is to provide a general understanding of the ways in which realistic flow conditions can alter larval dispersal kernels and levels of connectivity along the coast (see 'Discussion'). Drifters were therefore tracked for $30 \mathrm{~d}$, at which time they were considered to have successfully settled on the coast if their final position was located within $1 \mathrm{~km}$ of the coast.

Analysis of results. Although drifters were released along the entire coast within the model domain, we concentrated on the region between Punta Toro (Location 13) and Curaumilla (Location 28). Additionally, only Locations 10 to 22 were analysed as sources, and Locations 13 to 28, as sinks. Release locations outside these bands were treated only as sources or sinks for the central section of the coast, as their proximity to the boundary distorted their dispersal statistics. Empirical dispersal kernels were then constructed by binning the final along-shore position of the drifters, with bins of width $5 \mathrm{~km}$ centred on each release location. A Kolmogorov-Smirnov goodness-of-fit test was used to 
determine whether the larval distributions were consistent with random sampling from a normally distributed population. In general, all hypothesis testing follows a $5 \%$ significance level unless otherwise stated.

In a similar way to which the dispersal kernel $K_{x}$ predicts the average destination of larvae from a given release location $x$ (their destination sink), we defined an accumulation kernel $L_{x}$ which predicts the larval source locations $x^{\prime}$ of competent larvae settling at $x$.

That is:

$$
L_{X}\left(x^{\prime}\right)=\frac{\text { larvae arriving from } x^{\prime}}{\text { larvae settled at } x}
$$

Clearly then, the accumulation kernel at any point is a function of the dispersal kernels at every release location, viz.:

$$
L_{X}\left(x^{\prime}\right)=\frac{n_{X^{\prime}} K_{X^{\prime}}(x)}{\int_{-\infty}^{\infty} n_{X^{\prime \prime}} K_{X^{\prime \prime}}(x) \mathrm{d} x^{\prime \prime}}
$$

where $n_{X}$ represents the rate of larval release from Point $x$. The accumulation kernel may be a more significant distribution to consider than the dispersal kernel for net sinks of competent larvae, such as nursery grounds.

\section{RESULTS}

Both total larval distribution (shaded bars in Fig. 3a) and larval settling (effective dispersal kernel, open bars in Fig. 3a) statistics depart significantly from Gaussian, i.e. the larval dispersal distances do not represent a random sample from a normally distributed population. Rather, they have a leptokurtic distribution, such that the most likely displacement (the mode) is approximately $20 \mathrm{~km}$ less than the mean displacement in each case. The discrepancy between the dispersal distance and standard deviation between successful and non-successful larvae is related to the spatial variability in the flow shown in Fig. 4, in particular its cross-shore component. This is a non-trivial result with important implications for larval ecology that will be discussed later.

The mean, mode and standard deviation of the dispersal kernels for settling larvae as a function of release location each exhibit substantial along-shore variability over short spatial-scales (Fig. 3b, release locations are separated by $5 \mathrm{~km}$ in the along-shore direction). The difference between mean and mode of the kernels gives an indication of how leptokurtic the distribution is. The mode was less than the mean for all locations south of Location 21, indicating that the dispersal kernels are generally non-Gaussian, with their peak close to the origin and a long tail, similar to those in Fig. 3a. The mean and standard deviation of the dispersal kernels tend to become reduced to the north of Punta Toro, reaching a minimum value close to Las Cruces. This corresponds with the patterns of mean velocity and variance at each point in Fig. 4. It will be shown below that this spatial variability in the statistics of dispersal renders the spatially averaged kernel in Fig. 3a (or any other average kernel for the region with any shape) unable to predict important properties of settlement; therefore, it is unable to capture critical characteristics of connectivity among local subpopulations.

Similar variability in dispersal statistics was not seen in the time domain. Dispersal kernels averaged by month over the $4 \mathrm{yr}$ of the simulation and Release Locations 10 to 22 varied little, indicating a weak seasonal cycle (Fig. 3c). The kernel standard deviation appears to reach its maximum during austral winter and the minimum at the start of the austral summer, while little trend is evident in the mean. The winter variance maximum may result from the
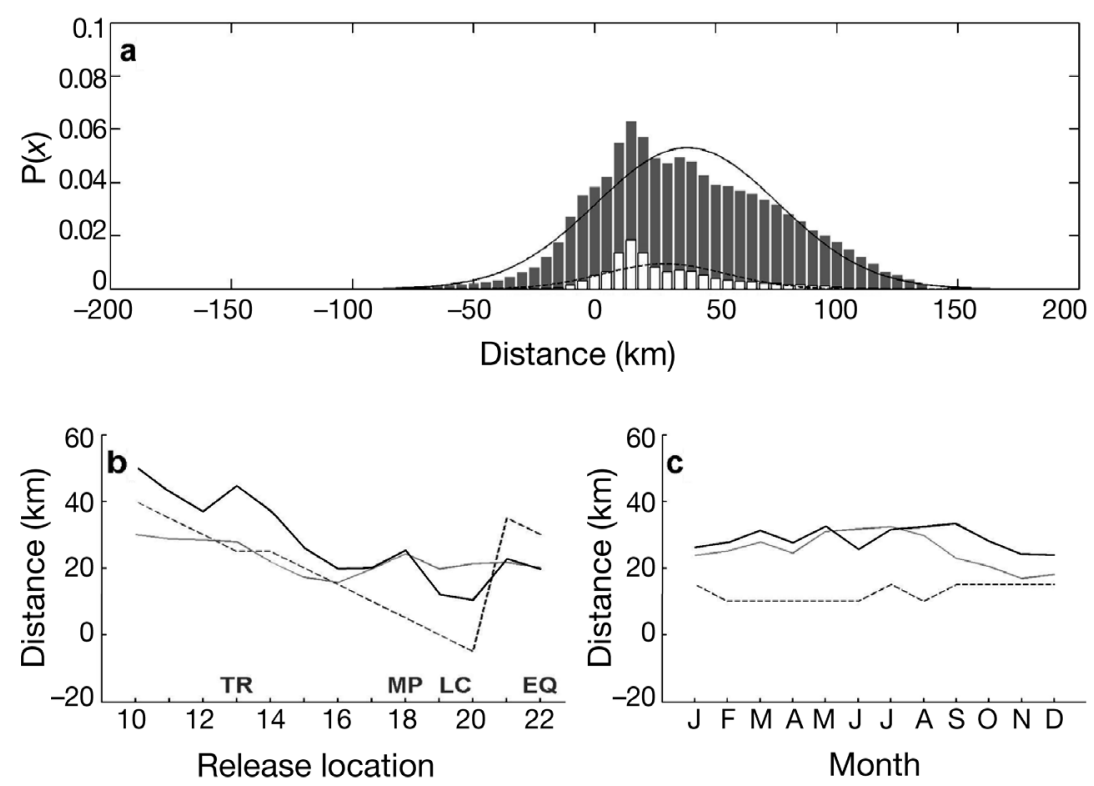

Fig. 3. (a) Probability of settling for all larvae released (shaded bars) and for those settling on the shore (open bars) as a function of along-shore distance and averaged over Release Locations 10 to 22 and all release times. The Gaussian distribution with the same mean and standard deviation for each are shown as the solid and dashed curves, respectively. Mode (dashed line), mean (solid dark line) and standard deviation (solid light line) of the dispersal kernel in kilometres as a function of (b) release location or (c) month. The approximate position of a number of the key locations from Fig. 1 are marked in Panel (b) for reference 

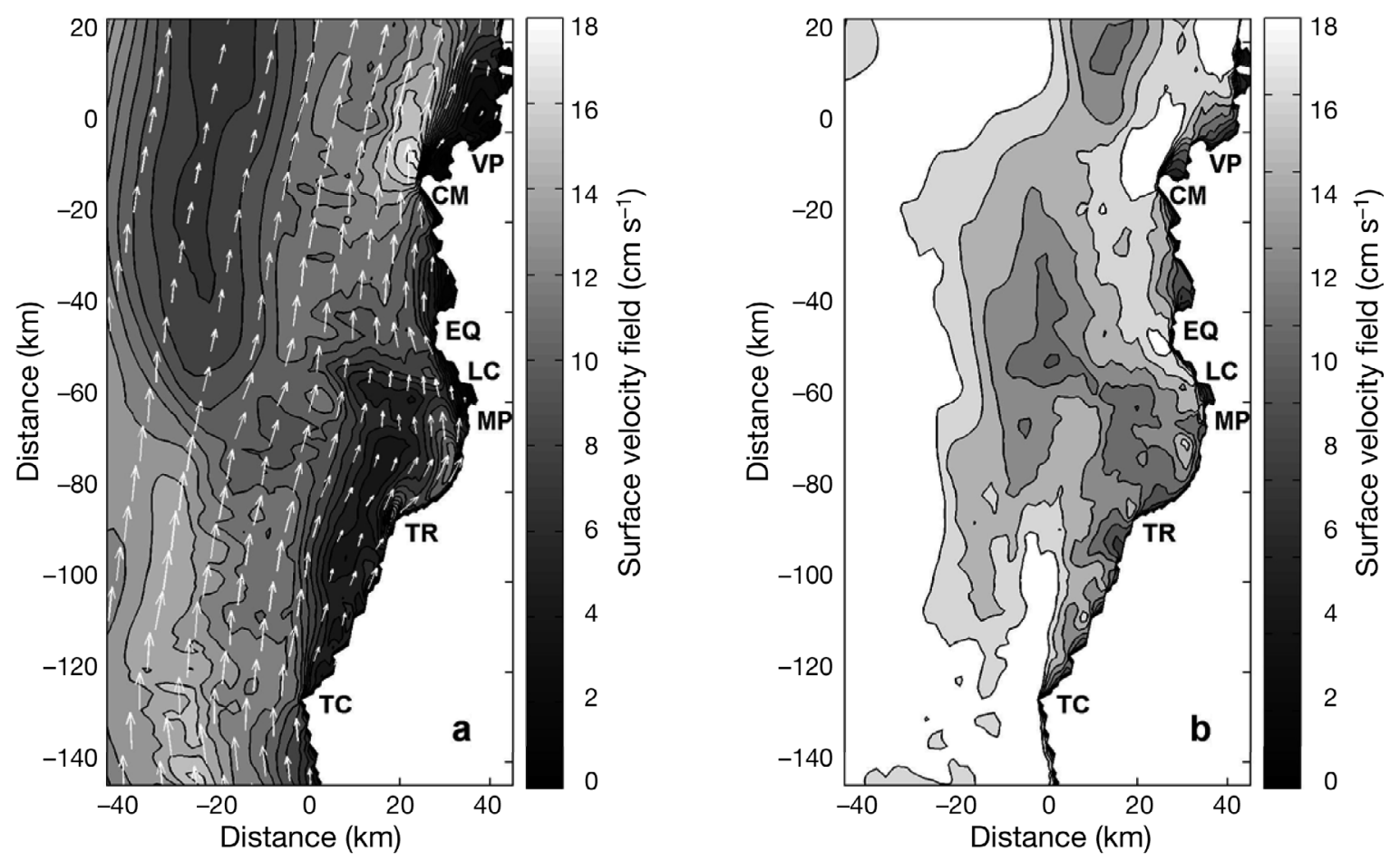

Fig. 4. (a) Mean and (b) standard deviation in the surface velocity field. In Panel (a) velocity magnitude is shaded and direction is indicated by vectors (site abbreviations, see Fig. 1)

occurrence of northerly wind events during winter months.

The fraction of larvae released from each location that successfully settle somewhere on the coast varied dramatically along-shore (Fig. 5a). Larvae released in the vicinity of Location 16 (from Punta Toro to the outfall of the Maipo River; Fig. 1) are the most likely to settle somewhere on the shore, while from Location 18 (Maipo River) to the north settling rates of released larvae are in general substantially lower. Location 16 is referred to hereafter as the 'maximum source'. The spatial distribution of settled larvae was calculated as a function of location by normalising the number of settled larvae within a distance of $1 \mathrm{~km}$ of each release location by the total number of larvae released from each location. Normalisation is done so that the quantity also corresponds to the input (sink) to output (source) ratio at each location. As was the case for the success rate of larval release locations, the percentage of larvae settling at each location along the coast varied strongly (Fig. 5b). In this case Location 19, hereafter called the 'maximum sink', was strongly favoured for settlement, with the number of released larvae settling within $1 \mathrm{~km}$ of this location being close to $10 \%$ of the number released from that and any location. Settlement south of the Maipo River (Location 18) was much less than that occurring to the north, although settling

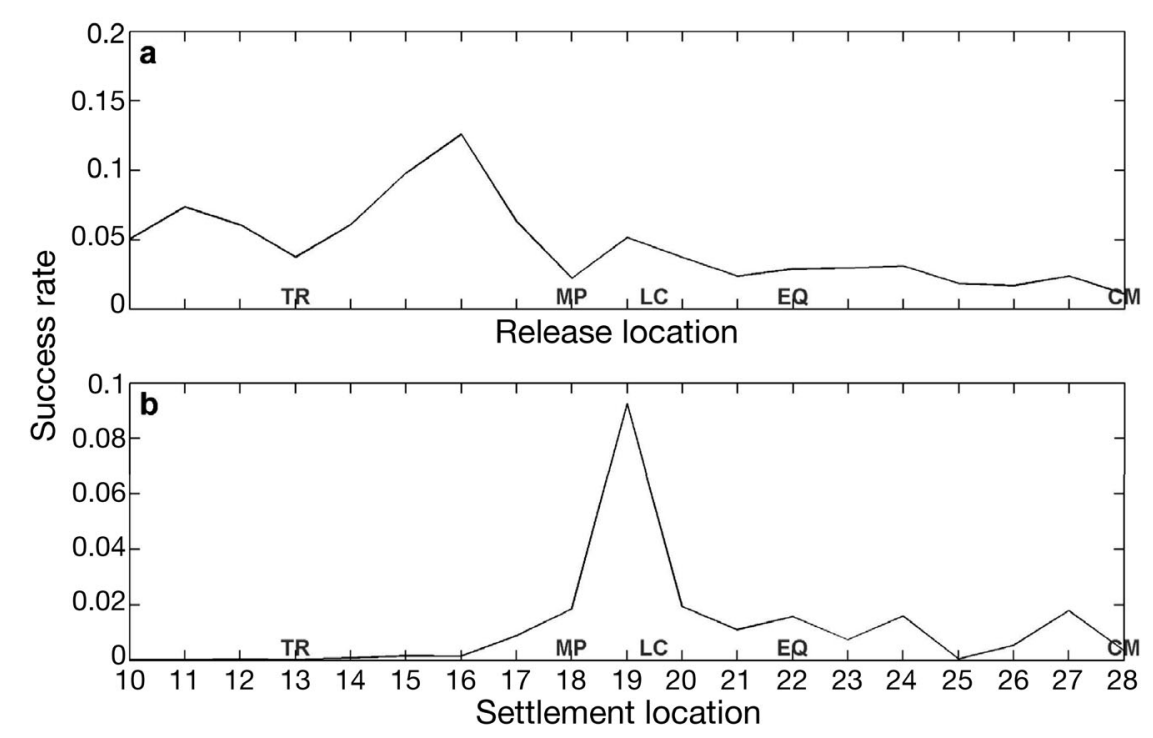

Fig. 5. Success rate of larval settlement as a function of (a) release location or (b) settlement location, averaged over the entire simulation (site abbreviations, see Fig. 1) 
larvae were found at all locations, even if in very low numbers. Thus, large and temporally persistent variability in larval sources/sinks results solely from the wind-driven ocean circulation.

If the dispersal kernel $K_{x}$ is independent of $x$, such as would be the case for a straight coastline and homogenous mean and variance of ocean velocity, and the larval production rate $n_{X}$ is also spatially uniform (as is the case in our model), then the shape of the dispersal and accumulation kernels $\left(L_{x}\right)$ will be identical. Yet, the time-averaged dispersal kernel for the 'maximum source' and accumulation kernel for the 'maximum sink' show important differences (Fig. 6). The vast majority of settled larvae released from the maximum
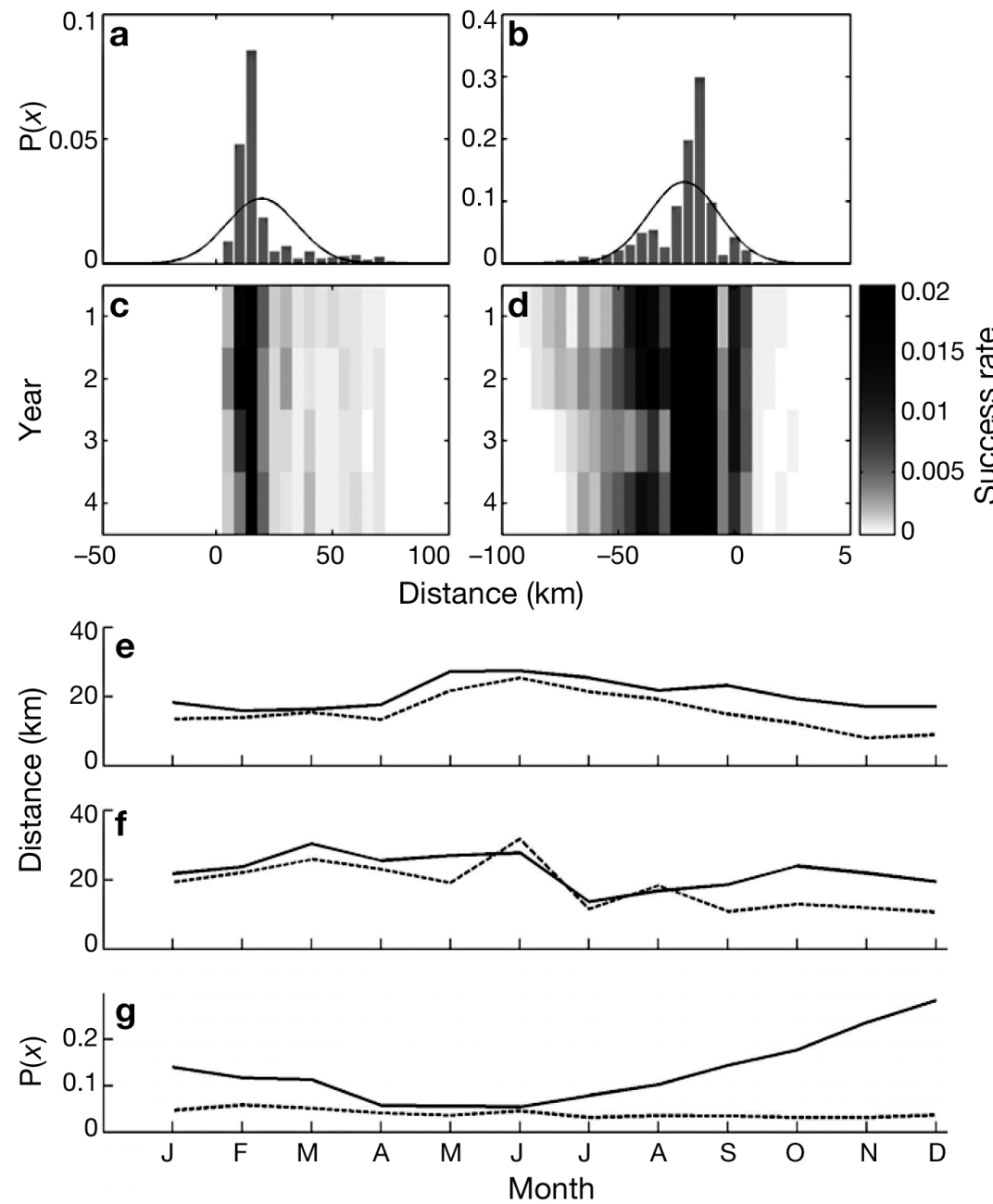

Fig. 6. (a) Dispersal kernel for maximum source (Location 16). (b) Accumulation kernel for maximum sink (Location 19). (c) Annual average success rate as a function of settlement position and year for the maximum source. (d) Annual average success rate as a function of release position and year. (e) Variability in mean (solid line) and standard deviation (dashed line) of the dispersal kernel of maximum source as a function of month. (f) As in Panel (e), but for the accumulation kernel of the maximum sink. (g) Success rate of larvae released from the maximum source (solid line) and from all locations (dashed line) as a function of month source were found between 10 and $20 \mathrm{~km}$ north (Fig. 6a). Very few larvae settled at the release point $(<1 \%$ self-recruitment) and few also settled north of El Quisco (EQ in Fig. 1b). The main sources of settling ettling larvae also arrived at the maximum sink from south of Punta Toro and a small amount from El Quisco to the north. That is, the maximum sink is more extensively connected than the maximum source. Although there is substantial quantitative annual variability in these kernels, their major features are seen to be qualitatively robust from year to year (Fig. 6c,d). The success of larval settlement at the maximum sink may be explained from the simulated velocity field (see 'Discussion'). Thus, the levels of larval connectivity, as well as the direction of the major larval flows among subpopulations, vary greatly and in a nonuniform way along the coast as a result of coastal circulation alone.

While the spatially averaged settlement rate shows little seasonal variability (Fig. 3), the success rate of larvae originating from the maximum source varies strongly during the year (Fig. 6g). Settlement success for larvae originating from this location was lowest during the early winter, when northerly winds are most common and southerlies weakest, resulting in more larvae being carried southward. Settlement of larvae released from the maximum source reaches a maximum during early austral summer, coincident with the period of strongest southerly winds (cf. Fig. 2). Of the larvae released from the maximum source that successfully settle, the majority settle at the maximum sink. This larval pathway appears to be most efficient during strong southerly winds. Although seasonality was weak in the dispersal kernel calculated by averaging all settlement data, a stronger annual cycle was apparent in the settlement characteristics associated with the main larval pathway between the maximum source and maximum sink. Mean and variance of the dispersal and accumulation kernels at these contrasting locations (source and sink, respectively) varied greatly as a function of month of the year (Fig. 6e,f), both reaching a 
maximum in austral winter and a minimum in austral summer. The summer minimum in dispersal distance from the maximum source coincides with the winter abatement of the south-westerly winds (Fig. 2), which could trap larvae near Las Cruces and hence limit dispersal. The picture is less clear in the case of the maximum sink, with both mean and standard deviation of its dispersal kernel being low from mid-winter to early summer. The dispersal kernel seasonal cycle at the maximum source is likely complicated by the fact that it is fed by a broader geographical range of sources, each of which may have slightly different seasonal dispersal characteristics.

\section{DISCUSSION}

We have investigated the statistics of passive dispersal of planktonic larvae released on-shore in a $4 \mathrm{yr}$ high resolution numerical simulation of the winddriven circulation on a section of the central Chilean coast. The numerical modelling approach used here and elsewhere appears to offer some advantages over parameterised models of dispersal for including realistic site-specific larval dispersal in studies of marine population dynamics. Coastal flows typically demonstrate high temporal and spatial dependence, while an important part of their variability may be correlated on larval advection time scales and, hence, cannot be represented by a stochastic process. Direct flow simulation is necessary for resolving many retention processes, such as topographic eddy generation or flow reversals (Cowen et al. 2000, 2006). While our model simulates flow and larval dispersal specifically for central Chile, topographically as well as in terms of major circulation patterns, this is not a particularly atypical coastline. Moreover, by modelling a coastally released, passive, neutrally buoyant planktonic larvae with a $30 \mathrm{~d}$ development time, the insights gained from our coupled biophysical model are not species specific and thus may shed light on coastal dispersal processes in general.

It should be borne in mind, however, that there are a number of factors not included in the model, such as tides, coastally trapped waves, and sub-grid scale turbulence, that would be expected to increase the realism of the model and could each potentially affect our results, possibly smoothing out the distributions or making them closer to Gaussian. For instance, inclusion of stochastic turbulence in numerical models has been shown to influence the dispersal of polychaete and fish larvae (Guizien et al. 2006). However, this approach requires an a priori choice of turbulence dispersal shape, which will, in turn, influence the resultant dispersal kernels. As a result we concentrate here on the dispersal due to wind-driven currents for which no a priori assumption of their statistics has to be made. Clearly no model is capable of simulating processes at all the scales that affect larval transport, from whitecaps to basin-scale circulation patterns, but important insights may still be gained into the effects of the modelled processes. In the case considered here, wind-driven flow is the dominant circulation component and, thus, predominantly determines passive larval dispersal in the real system.

Analysis of the modelled trajectories of larvae allowed estimation of the main dispersal routes and the degree of connectivity among local sub-populations. While, in general, sources are located to the south of sinks, as the prevailing currents encourage predominantly northward migration, southward migration was also possible, due to occasional reversals of the northward flow next to the coast. This raises the possibility that populations along the entire stretch of coast may be connected over time scales of years, although the time scales associated with northward and southward migration are likely to differ greatly. A dominant larval pathway was identified within the region, with larvae released on the stretch of coastline between Punta Toro and Maipo River having a relatively high likelihood of successfully settling in the vicinity of Las Cruces. In contrast, larvae released south of Punta Toro or north of El Quisco were found to have much less likelihood of settling anywhere on the coast. A number of coastal features appear to control connectivity in the model over shorter time scales. For example, populations south of Punta Toro are less connected to the Las Cruces area than those to the north. El Quisco also appears to divide locations that provide larvae to Las Cruces from those that do not. Similarly, larvae from Valparaíso Bay and to the north have difficulty in migrating south past Curaumilla. Such strong spatial asymmetry in larval connectivity about a given location can have important consequences for population genetic structure and models of genetic isolation by distance (Palumbi 1994). These complex patterns of along-shore variation in connectivity and settlement rates are not possible to elucidate using average dispersal kernels.

The patterns of settlement can be understood by considering the simulated ocean circulation. The orientation of the coastline and system of currents found between Las Cruces and the Maipo River under typical conditions seemed to conspire to trap larvae close to the coast and thus to allow increased settlement onshore. During the south-westerly winds that predominate in the region, the Las Cruces area lies in-shore of the northward-oriented coastal jet and contains a weak on-shore flow in which larvae from upstream sources were entrained and held close to shore. This 
local circulation is consistent with the existence of an upwelling shadow south of Las Cruces (Wieters et al. 2003, Narváez et al. 2004). Larvae from sources south of Punta Toro or north of Las Cruces, however, were more likely to be carried northwards in the coastal jet without striking land or being entrained in the coastal boundary layer; thus, these areas were less successful release locations. A spatially intensive barnacle recruitment study conducted in the same region during the spring to summer period found a remarkably similar pattern, with peak settlement and recruitment of 2 chthamalid barnacle species in the area of Las Cruces and low values around Punta Toro and Curaumilla (Lagos et al. 2005). Although suggestive, this coincidence in spatial patterns between our modelled larval dispersal and barnacle recruitment should be considered with caution, since barnacle larvae are not passive particles.

The seasonality observed in settlement success rate of the maximum source (Fig. 6) was related to the annual cycle in the wind field applied to force the model (Fig. 2). The dominant larval dispersal mechanism operated most effectively during late spring/early summer, a period associated with the most consistent south-westerly winds, which drove surface larvae into the Las Cruces upwelling shadow. That settlement elsewhere on the coast was subdued under the same conditions may be explained by the off-shore Ekman transport, greater cross-shore current variability and increased along-shore advection. As a result, settlement rates for many of the less successful locations reached their maxima during autumn/winter, opposite to the results for the maximum source and maximum sink. This result implies that, for species with extended reproduction and an average larval duration of $30 \mathrm{~d}$, coastal circulation alone could strongly influence the observed seasonal recruitment pattern and the degree of spatial synchrony in population dynamics, even between locations 10s of kilometres apart. During winter the northward jet was weaker and occasionally reversed during brief periods of northerly winds. Such conditions favour downwelling, causing on-shore flow, and thus would be expected to enhance settlement (Roughgarden et al. 1988). This appears to occur in some places on the coast, but not in the Las Cruces region, where the relatively low winter settlement rate indicates that such winds tend to flush out retained larvae to the south.

The differences in larval dispersal characteristics along-shore, cross-shore and as a function of season, which are observed in the model, suggest that a single representative dispersal kernel may be of limited use in specific situations. The importance of accounting for the large spatial and temporal variability in dispersal dynamics has been discussed previously (Lockwood et al. 2002, Kaplan 2006). The dispersal kernel formed by averaging all drifter dispersal data spatially and temporally (Fig. 2a) corresponds reasonably well with scales for the mean and the variability of ocean flow. The same was not true, however, for the kernel formed only by larvae that successfully settled. If the mean and variability in the modelled ocean velocity field were spatially homogenous, the distributions in Fig. 2a would be statistically indistinguishable. However, the hypothesis that both histograms were sampled from the same population can be rejected, suggesting that successful larvae experience a distinct ocean velocity field to unsuccessful larvae (Pineda 2000). This implies that larvae could potentially select oceanic environments, through mechanisms such as behavioural vertical migration, lee-side eddy traps, or acoustic, olfactory guidance. This implies selection pressure for reduced dispersal at the time of hatching, rather than selection of on-shore transport at the time of competency. The latter would lead to the same type of settlement distribution resulting from a random sample of a well-mixed larval pool. Larvae removed from the coastal 'boundary layer' into the stronger mean flow off-shore travel further, spread more and are less likely to be able to return to the coast to settle within the competency time window. This result is consistent with the propositions of Largier (2003), who discusses the need for careful choice of advective and diffusive scales based, in part, upon the length of time larvae are located within the coastal boundary layer and challenges the idea of a homogenous larval pool. It should be investigated whether similar patterns are observed for deeper water species that release larvae farther off-shore. We can speculate from our model that dispersal spread should be larger for off-shore species and that retention within a given geographic area might require very specific larval behaviours.

Recognising that the Gaussian dispersal kernel might not provide a good representation of actual larval dispersal (Lockwood et al. 2002, Chesson \& Lee 2005), several authors have investigated the effects of different shapes of dispersal on marine reserve design, assuming a constant (Lockwood et al. 2002) or temporally variable (Kaplan 2006) dispersal environment. The general conclusion from these studies is that the shape of the dispersal kernel has minor effects on connectivity and decisions about size or spacing of reserves. While these general conclusions can certainly help guide primary plans for conservation and fisheries management, specific reserve design over a real coastline might benefit from a spatially explicit approach such as the one followed here. Indeed, although our model does not consider the adult stage of the populations, results suggest that even with adequate estimation of ocean transport scales, an aver- 
aged dispersal kernel would be a poor model of dispersal and settlement and therefore connectivity among individual release locations. For the constant alongshore larval production assumed here, a spatially independent dispersal pattern would result in constant settlement rates along the coast. Instead, substantial spatial variability was seen in the characteristics of dispersal and settlement, reflecting the fact that ocean current characteristics and coastal geometry change significantly along-shore. With such variability on spatial scales less than that associated with larval advection, a dispersal kernel formed by global averaging loses important location-specific dispersal characteristics. It has been found here that processes occurring close to the coast are most important for determining the ability of larvae to settle. These local coastal flows typically have higher spatial variability than flow further off-shore, thus making it important that local flow characteristics be resolved accurately through observations or numerical simulation in order to understand larval dispersal processes.

Largier (2003) argues the utility of characterising oceanic dispersal using 2 parameters: mean flow and variance. While this is a large improvement over models that use just 1 parameter (or none), clearly it is not possible to describe dispersal adequately only with global estimates of advective and diffusive scales. A spatially independent advection-diffusion model results in Gaussian distributions, while the dispersal kernels calculated here and elsewhere are, in general, significantly non-Gaussian. At least a third parameter, and potentially many more including both physics and larval behaviour, would be necessary to adequately approximate the dispersal kernel's shape and, thus, to capture details of connectivity and source-sink dynamics along the coast. Unlike the case for defining a Gaussian kernel, it is not obvious whether or how these higher moments can be suitably estimated from the ocean velocity field. Even for Gaussian kernels, estimation of advective and diffusive scales is non-trivial, as it was found here that the velocity fields experienced by settling and non-settling larvae were statistically distinct. Thus, although the simple advectiondiffusion model of dispersal provides an efficient and useful approximation, this study suggests that greater realism in effective larval dispersal along the shore, and especially when evaluating the effectiveness of a marine reserve network in conservation and management, may be achieved through direct numerical simulation. Indeed, better larval dispersal modelling is vital for improving the design of marine reserve and fisheries management (Gaines et al. 2003). Our results suggest that numerical modelling may be able to capture the essence of average larval dispersal as well as along-shore variation in sources and sinks of larvae for the regional metapopulation. The approach is thus promissory to investigate the effectiveness of marine protected areas.

Acknowledgements. We thank F. Tapia and B. Broitman for comments and discussions on this study. Four anonymous reviewers provided cogent suggestions and criticism that substantially improved presentation. This research was supported by an Andrew Mellon Foundation grant to S.A.N. and J.C.C. and by FONDAP-FONDECYT Grant 15001-001 to the Center for Advanced Studies in Ecology and Biodiversity. Additional support while writing this contribution was provided by Fondecyt Grant No. 1040787 to S.A.N.

\section{LITERATURE CITED}

Botsford LW, Moloney CL, Hastings A, Largier JL, Powell TM, Higgins K, Quinn JF (1994) The influence of spatially and temporally varying oceanographic conditions on meroplanktonic metapopulations. Deep-Sea Res 41:107-145

Chesson P, Lee CT (2005) Families of discrete kernels for modeling dispersal. Theor Popul Biol 67:241-256

Cowen RK, Lwiza KM, Sponaugle S, Paris CB, Olson DB (2000) Connectivity of marine populations: Open or closed? Science 287:857-859

Cowen R, Paris CB, Srinivasan A (2006) Scaling of connectivity in marine populations. Science 311:522-527

Gaines SD, Gaylord B, Largier JL (2003) Avoiding current oversights in marine reserve design. Ecol Appl (Suppl) 13: $32-46$

Gardener CW (1985) Handbook of stochastic methods. Springer-Verlag, Berlin

Gerber LR, Botsford LW, Hastings A, Possingham HP, Gaines SD, Palumbi SR, Andelman S (2003) Population models for marine reserve design: a retrospective and prospective synthesis. Ecol Appl (Suppl) 13:47-64

Guizien K, Brochier T, Duchêne JC, Koh BS, Marsaleix P (2006) Dispersal of Owenia fusiformis larvae by winddriven currents: turbulence, swimming behaviour and mortality in a three-dimensional model. Mar Ecol Prog Ser 311:47-66

Hamrick JM (1992) A three-dimensional environmental fluid dynamics computer code: theoretical and computational aspects. Spec Rep Va Inst Mar Sci 317:1-63

Kaplan DM (2006) Alongshore advection and marine reserves: consequences for modeling and management. Mar Ecol Prog Ser 309:11-24

Kaplan DM, Largier JL, Navarrete SA, Guiñez R, Castilla JC (2003) Large diurnal temperature fluctuations in the nearshore water column. Estuar Coast Shelf Sci 57: 385-398

Kinlan BP, Gaines SD (2003) Propagule dispersal in marine and terrestrial environments: a community perspective. Ecology 84:2007-2020

Lagos N, Navarrete SA, Véliz F, Masuero A, Castilla JC (2005) Meso-scale spatial variation in settlement and recruitment of intertidal barnacles along the coast of central Chile. Mar Ecol Prog Ser 290:165-178

Largier JL (2003) Considerations in estimating larval dispersal distances from oceanographic data. Ecol Appl (Suppl) 13:71-89

Levin LA (2006) Recent progress in understanding larval dispersal: new directions and digressions. Integr Comp Biol 46:282-297 
Lockwood DR, Hastings A, Botsford LW (2002) The effects of dispersal patterns on marine reserves: Does the tail wag the dog? Theor Popul Biol 61:297-309

Narváez DA, Poulin E, Leiva G, Hernández E, Castilla JC, Navarrete SA (2004) Seasonal and spatial variation of nearshore hydrographic conditions in central Chile. Cont Shelf Res 24:279-292

Narváez DA, Navarrete SA, Largier J, Vargas CA (2006) Onshore advection of warm water, larval invertebrate settlement, and relaxation of upwelling off central Chile. Mar Ecol Prog Ser 309:159-173

Palumbi SR (1994) Genetic divergence, reproductive isolation, and marine speciation. Annu Rev Ecol Syst 25: $547-572$

Pineda J (2000) Linking larval settlement to larval transport: assumptions, potentials and pitfalls. Oceanogr E Pacif 1: 84-105

Piñones A, Valle-Levinson A, Narváez DA, Vargas CA, Navarrete SA, Yuras G, Castilla JC (2005) Wind-induced diurnal variability in river plume motion. Estuar Coast Shelf Sci 65:513-525

Editorial responsibility: Howard Browman (Associate Editorin-Chief), Storebø, Norway
Poulin E, Palma AT, Leiva G, Narváez D, Pacheco R, Navarrete SA, Castilla JC (2002) Avoiding offshore transport of competent larvae during upwelling events: the case of the gastropod Concholepas concholepas in central Chile. Limnol Oceanogr 47:1248-1255

Roberts CM (1997) Connectivity and management of Caribbean coral reefs. Science 278:1454-1457

Roughgarden J, Gaines SD, Possingham HP (1988) Recruitment dynamics in complex life cycles. Science 241: 1460-1466

Siegel DA, Kinlan BP, Gaylord B, Gaines SD (2003) Lagrangian descriptions of marine larval dispersion. Mar Ecol Prog Ser 260:83-96

Strub PT, Mesías JM, Montecinos V, Rutlland J, Salinas S (1998) Coastal ocean circulation off western South America. In: Robinson AR, Brink KH (eds) The sea, Vol 11. J Wiley \& Sons, New York, p 273-313

Wieters EA, Kaplan DM, Navarrete SA, Sotomayor A, Largier J, Nielsen KJ, Véliz F (2003) Alongshore and temporal variability in chlorophyll a concentration in Chilean nearshore waters. Mar Ecol Prog Ser 249:93-105

Submitted: June 5, 2006; Accepted: November 19, 2006 Proofs received from author(s): May 3, 2007 